\title{
A putative role for the aryl hydrocarbon receptor $(A H R)$ gene in a patient with cyclical Cushing's disease
}

\author{
Sunita M. C. De Sousa ${ }^{1,2,3^{*}}$ (D), Jim Manavis ${ }^{3}$, Jinghua Feng ${ }^{4,5}$, Paul Wang ${ }^{4}$, Andreas W. Schreiber ${ }^{4,5,6}$,
} Hamish S. Scott $2,3,4,5,6$ and David J. Torpy ${ }^{1,3}$

\begin{abstract}
Background: Apart from PRKAR1A mutations in a subset of cyclical Cushing's syndrome due to primary pigmented nodular adrenocortical disease, the molecular basis of cyclical Cushing's syndrome has not been investigated. We speculated that cyclical Cushing's syndrome may be due to mutations in the clock genes that govern circadian rhythms, including the hypothalamic-pituitary-adrenal axis.

Case presentation: A 47-year-old man presented with mass effects from a sellar lesion. He was ultimately diagnosed with cyclical Cushing's disease due to a giant corticotrophinoma. We performed whole exome sequencing of germline and tumour DNA, SNP array of tumour DNA and tumour immunohistochemistry in order to detect variants in candidate circadian/pituitary-associated genes. We identified a rare germline missense variant in the aryl hydrocarbon receptor (AHR) gene, which has previously been indirectly linked to pituitary tumorigenesis and clock system disruption. The AHR variant was found in a highly conserved site involved in phosphorylation. It was predicted to be damaging by multiple in silico tools and AHR tumour immunohistochemistry demonstrated loss of the normal nuclear staining pattern, suggestive of an inactivating mutation. We also found a novel, damaging germline missense variant in the retinoid $X$ receptor gamma ( $R X R G$ ) gene, multiple somatic chromosomal gains (including $A H R$ ), and a somatic mutational signature consistent with oncogenesis that may have acted synergistically with the AHR variant.

Conclusions: This is the first report of an AHR variant with predicted pathogenicity in the pituitary adenoma setting. Our preliminary data suggest that the highly conserved AHR gene may represent a link between pituitary tumorigenesis, the hypothalamic-pituitary-adrenal axis and the clock system. Further research may indicate a role for the gene in the development of cyclical Cushing's disease.
\end{abstract}

Keywords: Cyclical Cushing's, Aryl hydrocarbon receptor, Retinoid X receptor gamma, Whole exome sequencing, Clock genes

\section{Background}

Cyclical Cushing's syndrome (CCS), characterised by intermittent biochemical hypercortisolism, accounts for approximately $20 \%$ of endogenous Cushing's syndrome (CS) [1]. Cycles may last days to years, often with intraindividual consistency [1], suggesting an intrinsic fault in

\footnotetext{
* Correspondence: Sunita.DeSousa@sa.gov.au

${ }^{1}$ Endocrine and Metabolic Unit, Royal Adelaide Hospital, Adelaide, Australia

${ }^{2}$ Department of Genetics and Molecular Pathology, Centre for Cancer

Biology, an SA Pathology and University of South Australia alliance, Adelaide, Australia

Full list of author information is available at the end of the article
}

timekeeping. Competing theories for the pathogenesis of CCS include: episodic haemorrhage; periodic growth/ death of tumour cells; persistence of negative feedback; and, in cyclical Cushing's disease (CCD) only, altered hypothalamic control of the pituitary, via dopaminergic fluctuations for example [1].

The only known genetic cause of CCS is germline PRKAR1A mutations causing Carney's complex, including the common manifestation of ACTH-independent CCS due to primary pigmented nodular adrenocortical disease (PPNAD) [2]. However, PPNAD-associated CS

(c) The Author(s). 2020 Open Access This article is distributed under the terms of the Creative Commons Attribution 4.0 International License (http://creativecommons.org/licenses/by/4.0/), which permits unrestricted use, distribution, and reproduction in any medium, provided you give appropriate credit to the original author(s) and the source, provide a link to the Creative Commons license, and indicate if changes were made. The Creative Commons Public Domain Dedication waiver (http://creativecommons.org/publicdomain/zero/1.0/) applies to the data made available in this article, unless otherwise stated. 
may be either cyclical or non-cyclical [2], and cyclicity is thus not necessarily explained by PRKAR1A. The molecular basis of CCS has not otherwise been investigated. We performed whole exome sequencing (WES) in a man with CCD to investigate the possibility that CCS may be due to perturbation in the clock genes responsible for circadian rhythms, including the hypothalamicpituitary-adrenal (HPA) axis.

\section{Case presentation}

A 47-year-old man was found to have optic disc swelling by his optometrist. MRI revealed a $7.1 \mathrm{~cm}$ sellar mass (Additional file 1: Figure S1), shown to be an ACTH-positive pituitary adenoma on transsphenoidal biopsy. He had a history of obesity, hypertension, gout and renal calculi but no cyclical symptoms or blood pressure fluctuations. Body mass index was $52.1 \mathrm{~kg} / \mathrm{m}^{2}$ but he had no supraclavicular fat pads, Cushingoid striae, facial plethora, ecchymoses or proximal weakness. He had a right-sided oculomotor nerve palsy and right-sided proptosis and conjunctival injection suggesting ophthalmic vein compression. He was eupituitary apart from fluctuating ACTH-dependent cortisol production ranging from normal to 35 -fold ULN (Additional file 1: Figure S1). He was diagnosed with CCD due to a giant corticotrophinoma with intermittent biochemical hypercortisolism, although the precise temporal cyclicity could not be defined prior to transcranial partial tumour resection 1 week later. Histopathology confirmed a corticotrophinoma with no significant mitotic activity and a Ki67 count of < $1 \%$. He was eucortisolaemic immediately pre- and postoperatively with ACTH lowering from $376 \mathrm{ng} / \mathrm{L}$ (ULN 60) to $169 \mathrm{ng} / \mathrm{L}$ (Additional file 1: Figure S1). Postoperative complications included acute kidney injury, transient hyperglycaemia, pneumonia, deep vein thrombosis and central hypothyroidism. He later noticed improved BP control, reduced appetite and improved satiety with early but transient weight loss. Serial MRI showed a stable $4.2 \mathrm{~cm}$ tumour remnant (Additional file 1: Figure S1). Despite having typical CS comorbidities and postoperative complications, he has had no cyclical symptoms to guide the timing of investigations and no further episodes of overt hypercortisolism have been detected during intermittent testing. His family history is negative for endocrine tumours.

We performed WES of germline and tumour DNA and single nucleotide polymorphism (SNP) array of tumour DNA to identify sequencing variants and copy number variation in circadian/pituitary-associated genes. Tumour immunohistochemistry was performed to further evaluate the leading genetic variant of interest. Further details are provided in Additional file 1: Supplementary Methods.

\section{Results}

Germline DNA exhibited 14 rare, possibly damaging sequence variants in circadian/pituitary-associated genes (Additional file 1: Table S1). Tumour DNA did not exhibit any additional variants. Amongst the 14 germline variants of interest, only one variant was considered to be reliable and relevant to both circadian rhythm and pituitary tumorigenesis. This germline exon $10 A H R$ variant (GRCh37/hg19, Chr7:g.17379197C > T; ENST00000242057; c. $1748 \mathrm{C}>\mathrm{T} / \mathrm{p}$.Thr583Met) was present in the heterozygous state in both germline DNA (30/65 reads) and tumour DNA (86/177 reads). The variant is located in a site that is highly conserved and likely to be involved in phosphorylation [3] (Fig. 1). It was predicted to be damaging by five of six in silico tools. ExAC population allele prevalence is $0.01 \%$ with no homozygotes. It has been cited in Catalogue of Somatic Mutations in Cancer (COSMIC; cancer.sanger. ac.uk) in oesophageal adenocarcinoma [5]. Tumour immunohistochemistry showed restriction of AHR staining to the cytoplasm, whereas both cytoplasmic and nuclear AHR staining was seen in corticotrophinoma specimens from two male patients who had non-cyclical Cushing's disease and no AHR variants on WES (Fig. 2). By contrast, staining for the AHR chaperone, AIP, showed cytoplasmic and membranous staining in all three corticotrophinomas.

WES also revealed a novel, damaging heterozygous germline $R X R G$ variant (GRCh37/hg19, Chr1:g. 165379996C > T; ENST00000359842; c.856C > T/p.Arg286Cys) situated in the ligand binding domain (Fig. 3).

CNV analysis of tumour WES data revealed multiple chromosomal gains involving Chr 5,7,8,12-14,16,18-22 (Additional file 1: Figure S2). Orthogonal validation by SNP array showed $\operatorname{arr}(3,5,7) \times 3,(8) \times 4,(12,13,14) \times 3,(16) \times 4,(18$, $19,20,21,22) \times 3$. Involvement of Chr 7 by both WES and SNP array results indicated $A H R$ copy number gain in the tumour, whilst the two different ploidy counts with Chr 8 , 16 tetrasomy and Chr 3,5,7,12-14,18-22 trisomy by SNP array suggested the possibility of multiple tumour clones. Tumour DNA disclosed 20 high confidence variants with a predominant mutational signature (Additional file 1: Figure S3) matching that seen in most cancer types [7].

\section{Discussion}

The aryl hydrocarbon receptor (AHR) exists in a cytoplasmic complex with aryl hydrocarbon interacting protein (AIP), heat shock protein 90 and p23 protein [8]. Amongst many exogenous carcinogenic AHR ligands, the most potent is 2,3,7,8 tetrachlorodibenzo- $p$-dioxin (TCDD; dioxin) [9]. Activation by such ligands causes dissociation and nuclear translocation of AHR, followed by heterodimerisation with Ah receptor nuclear 


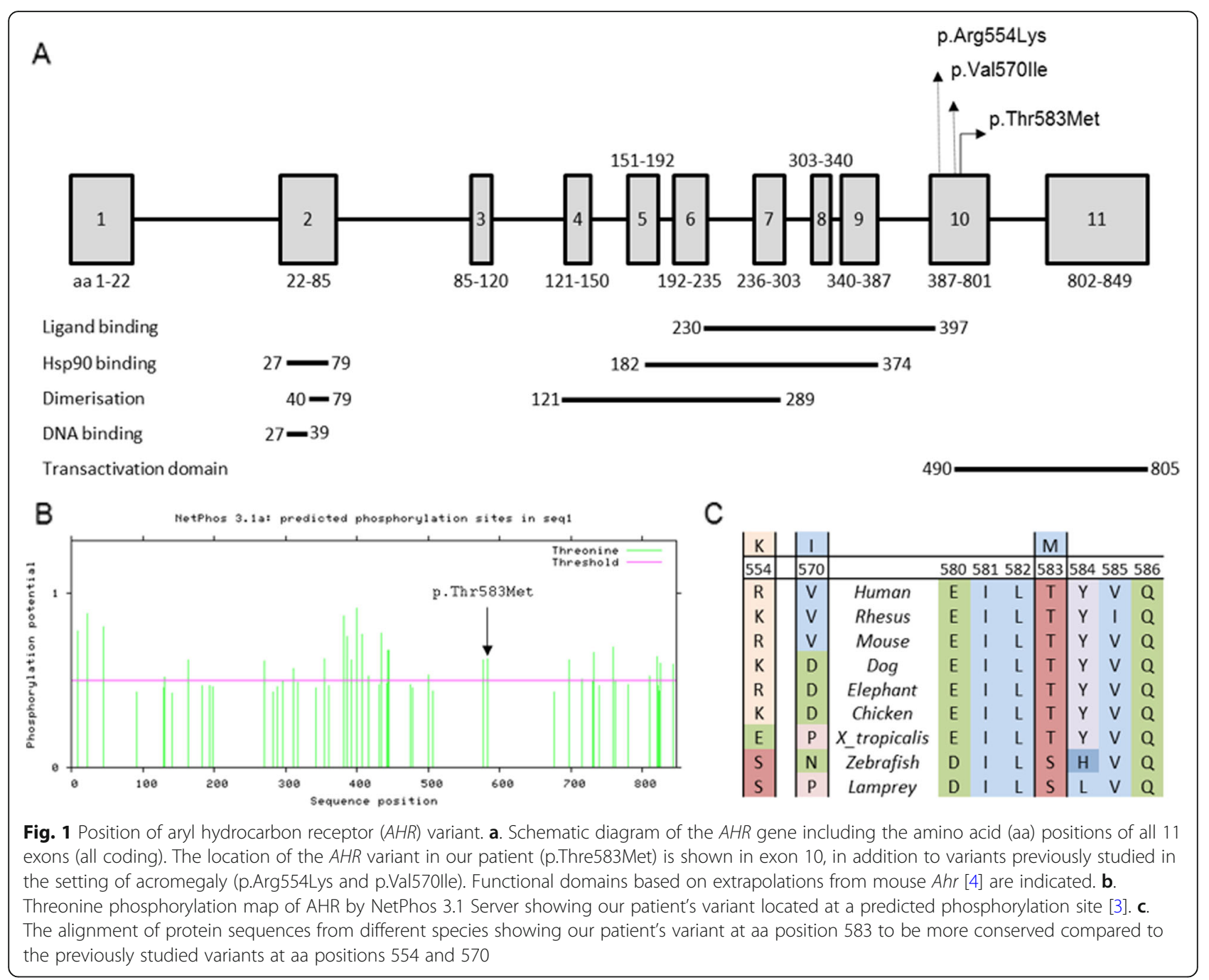

translocator (ARNT) and transcription of target genes involved in the cell cycle and clock system [8-11]. In the present study, we identified a germline Thr583Met $A H R$ variant in a man with $C C D$. We speculate that this likely inactivating variant may have contributed to CCD development via a loss of tumour suppressor function in the pituitary and disruption of circadian/infradian rhythms.

To the best of our knowledge, $A H R$ mutations have not been reported in patients with pituitary adenomas, including recent WES cohort studies of patients with Cushing's disease $[12,13]$. However, other lines of evidence support a tumour suppressor role for $A H R$ in the pituitary. AHR is the major binding partner of $A I P$, which is an established pituitary tumorigenesis gene [10, 11]. Loss of AHR stabilisation is postulated to be contributory to $A I P$-associated pituitary tumorigenesis, with somatotrophinomas from patients with germline AIP mutations typically showing decreased cytoplasmic and absent nuclear AHR staining [11]. AHR may also have $A I P$-independent roles in pituitary tumorigenesis as
$A H R$ but not AIP expression is reduced in GNAS-mutated somatotrophinomas, and $A H R$ activation increases the transcription of $C D K N 1 B$, which is another tumour suppressor gene implicated in pituitary and other endocrine tumours [10].

Whereas AIP germline mutations are most classically associated with somatotrophinomas, AHR may be more relevant to corticotrophinomas as in our patient. AHR immunostaining is found in the corticotroph-rich pars intermedia and normal corticotrophs demonstrate nuclear AHR immunostaining representative of activated $A H R$ [11], although there have been no systematic AHR immunostaining studies of corticotrophinomas to date. Furthermore, pro-opiomelanocortin is overexpressed in mice and pituitary cell lines treated with the AHR ligand, dioxin [14].

The location of our patient's $A H R$ variant at a highly conserved phosphorylation site [3] supports the pathogenicity of this variant. Loss of the normal nuclear pattern of AHR tumour staining suggests that it is a loss-of-function variant causing failure of nuclear translocation. It is 


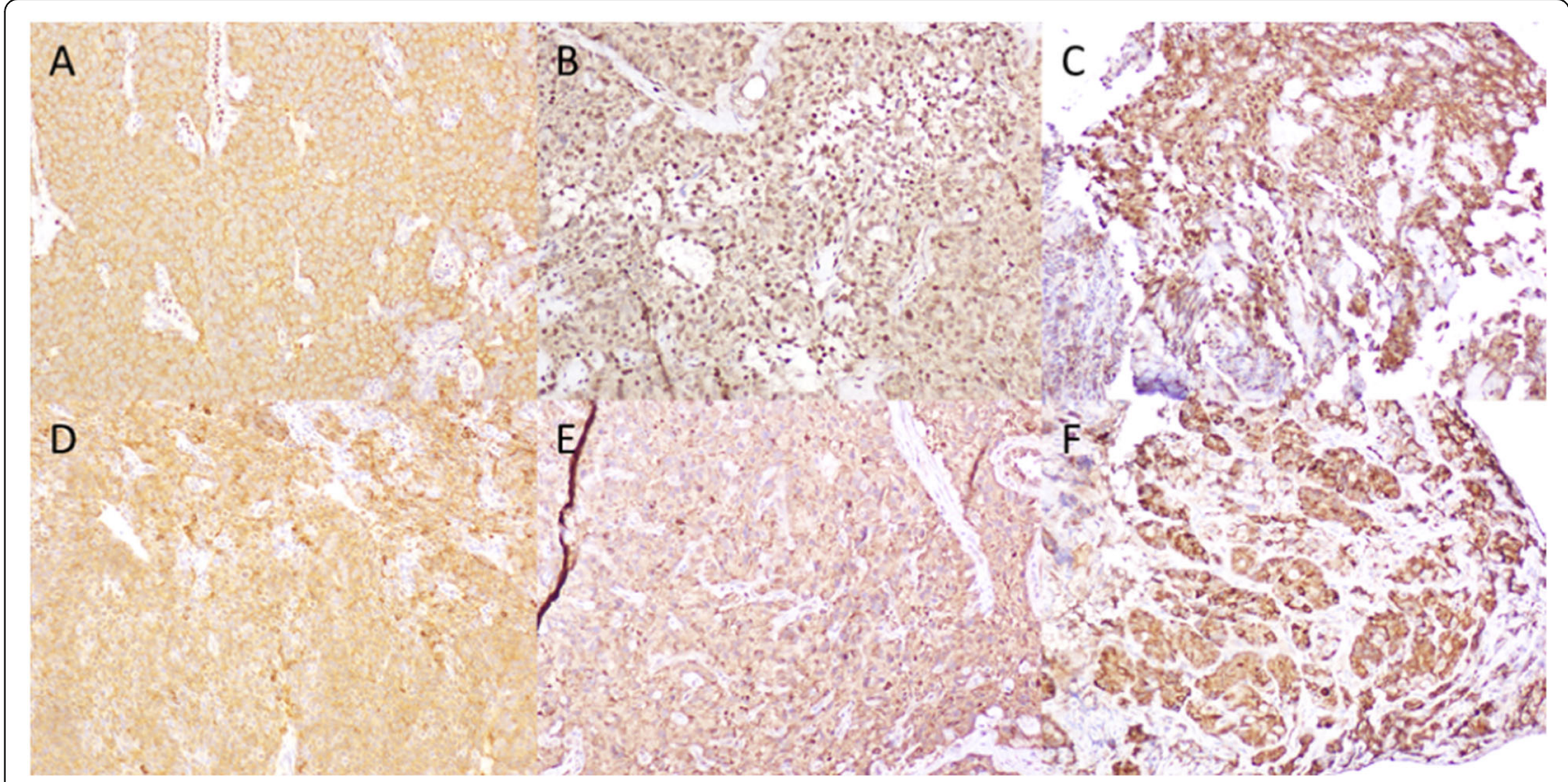

Fig. 2 AHR and AIP tumour immunohistochemistry (100x magnification). a. Only cytoplasmic AHR staining was observed in the patient's corticotrophinoma. b, c. Both cytoplasmic and nuclear AHR staining was observed in control corticotrophinoma specimens from two men with non-cyclical Cushing's disease and no AHR variants. d-f. Cytoplasmic and membranous staining for the AHR chaperone, AIP, was found in the corticotrophinomas from the patient (d) and the two controls (e, $\mathbf{f})$

possible that the somatic Chr 7 trisomy amplified a dominant negative effect of this variant by increasing mutant dosage in the tumour. However, other evidence suggests that $A H R$ may have proto-oncogenic effects. Recapitulating animal models, an excess of non-functioning pituitary adenomas (NFPA) and prolactinomas followed dioxin exposure from the 1976 Seveso accident in Italy [11], and acromegaly risk is 8-fold higher in Italian regions with high environmental exposure to AHR ligands such as cadmium [9]. The AHR SNPs, rs2066853 (c.1661G > A, p.Arg554Lys) and rs4986826 (c.1708G > A, p.Val570Ile), are over-represented in acromegalic patients in these regions with prevalences of 22.4 and $2.9 \%$, respectively, compared to Caucasian ExAC allele prevalences of 9.9 and $0.3 \%$, respectively [9]. Interestingly, these AHR SNPs and our patient's variant all reside in exon 10, encoding the transactivation domain (Fig. 1) [6]. Exon 10 SNPs are associated with other neoplasms, including glioma, but pituitary studies have been limited to acromegaly [9]. The differential tumour suppressor and protooncogenic effects of $A H R$ are yet to be fully elucidated but may depend on cell type.

$A H R$ has an additional emerging role in the clock system, which entrains sleep, appetite, metabolism, locomotion and reproductive activity to $24 \mathrm{~h}$ day-night cycles $[15,16]$. The upstream mediators of the clock system, circadian locomotor output cycle kaput (CLOCK) and brain-muscle-aryl hydrocarbon nuclear translocator-like protein 1 (BMAL1), heterodimerise and bind enhancer- box (E-box) regions in target genes, similarly to other members of the PER-ARNT-SIM (PAS) superfamily that includes AHR [15]. Separate to the canonical pathways of AHR/ARNT heterodimerisation and BMAL1/CLOCK heterodimerisation in the clock system, activated AHR can heterodimerise with BMAL1, indirectly affecting the regulation of diurnal patterns [16]. This is supported by greater amplitudes of downstream clock gene expression in Ahr-deficient versus wild-type mice [16]. On the other hand, pituitary adenomas are not reported in Ahrdeficient mice [16-18].

Other variants might have acted synergistically with the $A H R$ variant, particularly the novel germline $R X R G$ variant which has a Combined Annotation Dependent Depletion score of 34.0 (Additional file 1: Table S1). RXRG may act as a tumour suppressor gene in the pituitary as it is most highly expressed in the pituitary (GTEx; https://www.gtexportal.org/home/). Furthermore, RXRG belongs to the retinoid X nuclear receptor family that mediates the antiproliferative effects of retinoic acid, which has shown some efficacy in the treatment of Cushing's disease [19]. Another novel, likely damaging $R X R G$ variant (p.R317H) in the ligand binding domain segregated upon WES of a familial prolactinoma kindred [20], but RXRG has not previously been studied in Cushing's disease. Our patient also had a somatic mutational signature typical of various cancer types [7]. Though only 20 high confidence variants were available for the signature analysis, this is not uncommon in pituitary tumours $[21,22]$ and 


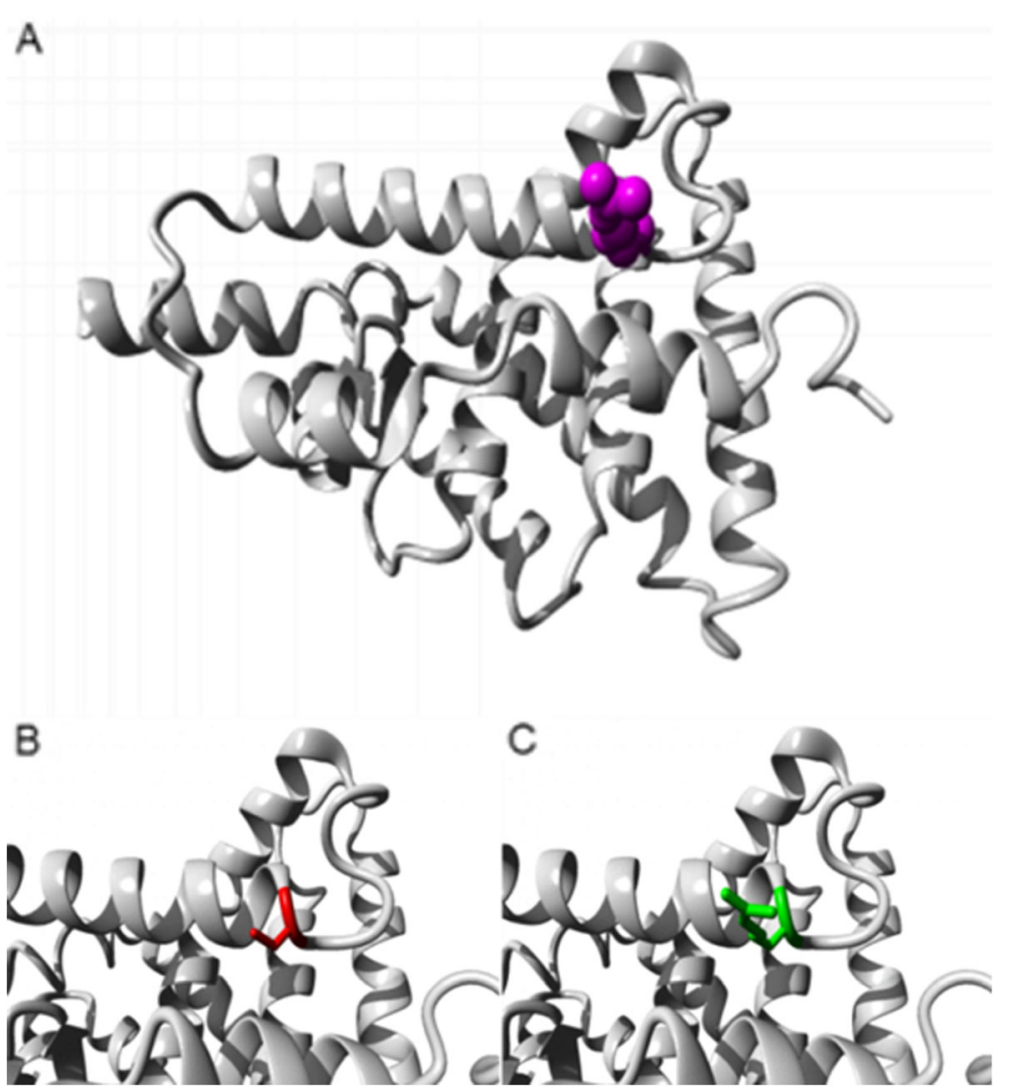

Fig. 3 Structure of human wild-type and mutant retinoid X receptor gamma (RXRG) protein [6]. a. Arg286 (magenta) is part of the ligand binding domain and forms a salt bridge with glutamic acid at position 241 and glutamic acid at position 282. b-c. The substitution Arg286Cys (red) was shown through homology modelling to be smaller than the wild-type Arg286 (green), which may cause loss of external interactions. The charge of the wild-type residue is also lost by this substitution, which will disturb the ionic interaction made by the wild-type residue with nearby glutamic acid residues

the signature found raises the possibility of cooperation between the germline $A H R$ and $R X R G$ variants and somatic driver mutations.

A limitation of this case study is that the patient is currently in a prolonged state of normocortisolism, precluding additional investigations to demonstrate ongoing cyclicity. This is despite a significant tumour remnant, highlighting the discordance between structural and functional status in patients with CCD. Given the rarity of Cushing's syndrome in general and CCD in particular, collaborative research is required to further examine the potential relationship between $A H R$ and CCD raised by this case study.

\section{Conclusions}

Preliminary data from this case study suggest that the highly conserved $A H R$ gene may represent a link between pituitary tumorigenesis, the HPA axis and the clock system, implicating it in the development of CCD. With $A H R$ known to be expressed in the pituitary, CCD may occur because of the combination of $A H R$-mediated pituitary tumorigenesis and disordered clock control of the HPA axis. Our patient's somatic Chr 7 trisomy and germline RXRG variant might have been additive to his germline $A H R$ variant, explaining how this variant can be seen in up to $1 / 10,000$ individuals in population data despite the rarity of CCD. Alternatively, $A H R$ variants might lead to cyclicity in individuals who happen to develop CS. Future research is required to determine whether $A H R$ is a true pituitary tumorigenesis gene or a phenotypic modifier gene accounting for cyclicity in CS of various aetiologies.

\section{Supplementary information}

Supplementary information accompanies this paper at https://doi.org/10. 1186/s12902-020-0495-8.

Additional file 1. Supplementary Methods, Figures and Table.

\section{Abbreviations}

AHR: Aryl hydrocarbon receptor; AIP: Aryl hydrocarbon interacting protein; ARNT: Ah receptor nuclear translocator; BMAL1: Brain-muscle-aryl hydrocarbon nuclear translocator-like protein 1; CCD: Cyclical Cushing's 
disease; CCS: Cyclical Cushing's syndrome; CLOCK: Circadian locomotor output cycle kaput; COSMIC: Catalogue of Somatic Mutations in Cancer: CS: Cushing's syndrome; E-box: Enhancer-box; GTEx: Genotype-Tissue Expression; HPA: Hypothalamic-pituitary-adrenal; NFPA: Non-functioning pituitary adenoma; PAS: PER-ARNT-SIM; PPNAD: Primary pigmented nodular adrenocortical disease; RXRG: Retinoid X receptor gamma; SNP: Single nucleotide polymorphism; TCDD: 2,3,7,8 tetrachlorodibenzo-p-dioxin; ULN: Upper limit of normal; WES: Whole exome sequencing

\section{Acknowledgements}

We thank neurosurgeon, Dr. Stephen Santoreneos for assisting in operative specimen collection and cytogeneticist, Dr. Sarah Moore for performing the SNP array.

\section{Authors' contributions}

DJT and SMCD conceptualised the study. SMCD analysed all data, performed protein modelling and wrote the manuscript. JM performed tissue immunohistochemistry. JF, PW and AWS processed raw WES data. HSS helped design the genetic investigations of the study, supervised all genetic investigations and assisted in data analysis. DJT is the patient's primary clinician. All authors read and approved the final manuscript.

\section{Funding}

SMCD is supported by an A.R. Clarkson Scholarship from the Royal Adelaide Hospital. The collection, analysis and interpretation of data were undertaken with support from a Royal Adelaide Hospital Project Grant, and with the financial and other support of the Cancer Council SA's Beat Cancer Project on behalf of its donors and the State Government of South Australia through the Department of Health.

\section{Availability of data and materials}

The datasets used and/or analysed during the current study are available from the corresponding author on reasonable request.

\section{Ethics approval and consent to participate}

The study was locally approved by the Central Adelaide Local Health Network Research Office (SSA/18/CALHN/445). The patient and control subjects provided written informed consent to participate.

\section{Consent for publication}

The patient and control subjects provided written informed consent for publication.

\section{Competing interests}

The authors declare that they have no competing interests.

\section{Author details \\ ${ }^{1}$ Endocrine and Metabolic Unit, Royal Adelaide Hospital, Adelaide, Australia. ${ }^{2}$ Department of Genetics and Molecular Pathology, Centre for Cancer Biology, an SA Pathology and University of South Australia alliance, Adelaide, Australia. ${ }^{3}$ School of Medicine, University of Adelaide, Adelaide, Australia. ${ }^{4}$ ACRF Cancer Genomics Facility, Centre for Cancer Biology, an SA Pathology and University of South Australia alliance, Adelaide, Australia. ${ }^{5}$ School of Pharmacy and Medical Sciences, University of South Australia, Adelaide, Australia. ${ }^{6}$ School of Biological Sciences, University of Adelaide, Adelaide, Australia.}

Received: 15 July 2019 Accepted: 20 January 2020

Published online: 29 January 2020

\section{References}

1. Meinardi JR, Wolffenbuttel BH, Dullaart RP. Cyclic Cushing's syndrome: a clinical challenge. Eur J Endocrinol. 2007;157(3):245-54.

2. Powell AC, Stratakis CA, Patronas NJ, Steinberg SM, Batista D, Alexander HR, et al. Operative management of Cushing syndrome secondary to micronodular adrenal hyperplasia. Surgery. 2008;143(6):750-8.

3. Blom N, Gammeltoft S, Brunak S. Sequence and structure-based prediction of eukaryotic protein phosphorylation sites. J Mol Biol. 1999;294(5):1351-62.

4. Fukunaga BN, Probst MR, Reisz-Porszasz S, Hankinson O. Identification of functional domains of the aryl hydrocarbon receptor. J Biol Chem. 1995; 270(49):29270-8.
5. Dulak AM, Stojanov P, Peng S, Lawrence MS, Fox C, Stewart C, et al. Exome and whole-genome sequencing of esophageal adenocarcinoma identifies recurrent driver events and mutational complexity. Nat Genet. 2013;45(5): 478-86.

6. Venselaar H, Te Beek TA, Kuipers RK, Hekkelman ML, Vriend G. Protein structure analysis of mutations causing inheritable diseases. An e-Science approach with life scientist friendly interfaces. BMC Bioinformatics. 2010;11:548.

7. Alexandrov LB, Nik-Zainal S, Wedge DC, Aparicio SA, Behjati S, Biankin AV, et al. Signatures of mutational processes in human cancer. Nature. 2013; 500(7463):415-21.

8. Formosa R, Borg J, Vassallo J. Aryl hydrocarbon receptor (AHR) is a potential tumour suppressor in pituitary adenomas. Endocr Relat Cancer. 2017;24(8): $445-57$.

9. Cannavo S, Ragonese M, Puglisi S, Romeo PD, Torre ML, Alibrandi A, et al. Acromegaly is more severe in patients with AHR or AIP gene variants living in highly polluted areas. J Clin Endocrinol Metab. 2016;101(4):1872-9.

10. Jaffrain-Rea ML, Rotondi S, Turchi A, Occhi G, Barlier A, Peverelli E, et al. Somatostatin analogues increase AIP expression in somatotropinomas, irrespective of Gsp mutations. Endocr Relat Cancer. 2013;20(5):753-66.

11. Jaffrain-Rea ML, Angelini M, Gargano D, Tichomirowa MA, Daly AF, Vanbellinghen JF, et al. Expression of aryl hydrocarbon receptor (AHR) and AHR-interacting protein in pituitary adenomas: pathological and clinical implications. Endocr Relat Canc. 2009;16(3):1029-43.

12. Chen J, Jian X, Deng S, Ma Z, Shou X, Shen Y, et al. Identification of recurrent USP48 and BRAF mutations in Cushing's disease. Nat Commun. 2018;9(1):3171.

13. Reincke M, Sbiera S, Hayakawa A, Theodoropoulou M, Osswald A, Beuschlein F. Mutations in the deubiquitinase gene USP8 cause Cushing's disease. Nat Genet. 2015;47(1):31-8.

14. Huang P, Ceccatelli S, Hakansson H, Grandison L, Rannug A. Constitutive and TCDD-induced expression of ah receptor-responsive genes in the pituitary. Neurotoxicology. 2002;23(6):783-93.

15. Nader N, Chrousos GP, Kino T. Interactions of the circadian CLOCK system and the HPA axis. Trends Endocrinol Metab. 2010;21(5):277-86.

16. Jaeger C, Khazaal AQ, Xu C, Sun M, Krager SL, Tischkau SA. Aryl hydrocarbon receptor deficiency alters circadian and metabolic rhythmicity. J Biol Rhythm. 2017:32(2):109-20

17. Lund AK, Goens MB, Kanagy NL, Walker MK. Cardiac hypertrophy in aryl hydrocarbon receptor null mice is correlated with elevated angiotensin II, endothelin-1, and mean arterial blood pressure. Toxicol Appl Pharmacol. 2003;193(2):177-87.

18. Vasquez A, Atallah-Yunes N, Smith FC, You X, Chase SE, Silverstone AE, et al. A role for the aryl hydrocarbon receptor in cardiac physiology and function as demonstrated by AhR knockout mice. Cardiovasc Toxicol. 2003;3(2):153-63.

19. Pecori Giraldi F, Ambrogio AG, Andrioli M, Sanguin F, Karamouzis I, Corsello SM, et al. Potential role for retinoic acid in patients with Cushing's disease. J Clin Endocrinol Metab. 2012;97(10):3577-83.

20. Melo FM, Couto PP, Bale AE, Bastos-Rodrigues L, Passos FM, Lisboa RG, et al. Whole-exome identifies RXRG and TH germline variants in familial isolated prolactinoma. Cancer Genet. 2016;209(6):251-7.

21. Bi WL, Horowitz P, Greenwald NF, Abedalthagafi M, Agarwalla PK, Gibson WJ, et al. Landscape of genomic alterations in pituitary adenomas. Clin Cancer Res. 2017;23(7):1841-51.

22. Song ZJ, Reitman ZJ, Ma ZY, Chen JH, Zhang QL, Shou XF, et al. The genome-wide mutational landscape of pituitary adenomas. Cell Res. 2016; 26(11):1255-9.

\section{Publisher's Note}

Springer Nature remains neutral with regard to jurisdictional claims in published maps and institutional affiliations. 